\title{
STRONG CONVERGENCE THEOREM FOR WALSH-KACZMARZ-FEJÉR MEANS
}

\author{
NATA GOGOLASHVILI, KÁROLY NAGY, GEORGE TEPHNADZE
}

\begin{abstract}
As main result we prove that Fejér means of Walsh-Kaczmarz-Fourier series are uniformly bounded operators from the Hardy martingale space $H_{p}$ to the Hardy martingale space $H_{p}$ for $0<p \leq 1 / 2$.
\end{abstract}

Key words and phrases: Walsh-Kaczmarz system, Fejér means, maximal operator, strong convergence, martingale Hardy space.

2010 Mathematics Subject Classification. 42C10.

\section{INTRODUCTION}

In 1948 S̆neider [26] introduced the Walsh-Kaczmarz system and showed that the inequality

$$
\limsup _{n \rightarrow \infty} \frac{D_{n}^{\kappa}(x)}{\log n} \geq C>0
$$

holds for the Dirichlet kernels $D_{n}^{\kappa}$ almost everywhere. In 1974 Schipp [17] and Young [32] proved that the Walsh-Kaczmarz system is a convergence system. Skvortsov in 1981 [24] showed that the Fejér means with respect to the Walsh-Kaczmarz system converge uniformly to $f$ for any continuous functions $f$. Gát [5] proved, for any integrable functions, that the Fejér means with respect to the Walsh-Kaczmarz system converge almost everywhere to the function. He showed that the maximal operator $\sigma^{\kappa, *}$ of Walsh-Kaczmarz-Fejér means is of weak type $(1,1)$ and of type $(p, p)$ for all $1<p \leq \infty$. Gát's result was generalized by Simon [20] (see also [22]), who showed that the maximal operator $\sigma^{\kappa, *}$ is of type $\left(H_{p}, L_{p}\right)$ for $p>1 / 2$.

In the endpoint case $p=1 / 2$ Goginava [8] proved that the maximal operator is not of type $\left(H_{1 / 2}, L_{1 / 2}\right)$. Moreover, in case $p=1 / 2$ Weisz [35] showed that the maximal operator is of weak type $\left(H_{1 / 2}, L_{1 / 2}\right)$. The investigation of the maximal operator $\sigma^{\kappa, *}$ was continued by Goginava and Nagy. In 2011 they proved that the maximal operator $\tilde{\sigma}^{\kappa, *}$ defined by

$$
\tilde{\sigma}^{\kappa, *}:=\sup _{n \in \mathbf{P}} \frac{\left|\sigma_{n}^{\kappa} f\right|}{\log ^{2}(n+1)}
$$

is bounded from the Hardy space $H_{1 / 2}$ to the space $L_{1 / 2}$ [9]. They also showed the sharpness of this result. Namely, they showed that for any nondecreasing function $\varphi: \mathbb{P} \rightarrow[1, \infty)$ satisfying the condition

$$
\varlimsup_{n \rightarrow \infty} \frac{\log ^{2}(n+1)}{\varphi(n)}=+\infty
$$

Research supported by projects TÁMOP-4.2.2.A-11/1/KONV-2012-0051, GINOP-2.2.1-15-2017-00055 and by Shota Rustaveli National Science Foundation grant no. FR-19-676. 
the modified maximal operator $\tilde{\sigma}_{\varphi}^{\kappa, *}:=\sup _{n \in \mathbb{P}} \frac{\left|\sigma_{n}^{\kappa} f\right|}{\varphi(n)}$ is not bounded from the Hardy space $H_{1 / 2}$ to the space $L_{1 / 2}$.

The case $0<p<1 / 2$ was studied by Tephnadze [27]. Namely, he showed that the maximal operator defined by

$$
\tilde{\sigma}_{p}^{\kappa, *}:=\sup _{n \in \mathbb{P}} \frac{\left|\sigma_{n}^{\kappa} f\right|}{(n+1)^{1 / p-2}}
$$

is bounded from the Hardy space $H_{p}(G)$ to the space $L_{p}(G)(0<p<1 / 2)$. He also showed that the sequence $(n+1)^{1 / p-2}$ is sharp.

In paper [28] (see also [15, 16, 29]) Tephnadze found necessary and sufficient conditions for the convergence of Walsh-Kaczmarz-Fejér means in the terms of modulus of continuity on the Hardy spaces $H_{p}$, when $0<p<1 / 2$ and $p=1 / 2$, separately. We note that the proofs of these two results based on the properties of the modified maximal operators $\tilde{\sigma}^{\kappa, *}$ and $\tilde{\sigma}_{p}^{\kappa, *}$ (for details see [9, 27]).

Simon [23] proved that there is an absolute constant $c_{p}$, depending only on $p$, such that

$$
\sum_{k=1}^{\infty} \frac{\left\|S_{k}^{\kappa} f\right\|_{p}^{p}}{k^{2-p}} \leq c_{p}\|f\|_{H_{p}}^{p}
$$

for all $f \in H_{p}(G)$, where $0<p<1$.

Similar problem for the Walsh-Kaczmarz system in the endpoint case $p=1$ is still open problem, but for the Walsh system analogue of this statement was proven in the work of Simon [21] (see also [2, 3, 6, 4, 31]) and for the trigonometric system it is proven by Smith [25].

In the present paper we prove for $0<p \leq 1 / 2$ and Walsh-Kaczmarz-Fejér means, that there exists a positive constant $c_{p}$ depending only on $p$, such that

$$
\frac{1}{\log ^{[p+1 / 2]} n} \sum_{m=1}^{n} \frac{\left\|\sigma_{m}^{\kappa}(f)\right\|_{H_{p}}^{p}}{m^{2-2 p}} \leq c_{p}\|f\|_{H_{p}}^{p}
$$

holds for all $f \in H_{p}$. Moreover, we show the sharpness of our main theorem. That is, we state a strong convergence result in the endpoint case $p=1 / 2$ which was investigated in papers [8, 9, 28, 35] and we prove a strong convergence result also in the case $0<p<1 / 2$ with related papers [27, 28]. We note that in 2014 analogical Theorems for Walsh-Paley system was reached by Tephnadze [30], the two-dimensional case was investigated by Nagy and Tephnadze [11, 12, 13].

Now, we give a brief introduction to the theory of dyadic analysis [1, 18]. Let $\mathbb{P}$ denote the set of positive integers, $\mathbb{N}:=\mathbb{P} \cup\{0\}$. Let $G$ be the Walsh group. The measure on $G$ is denoted by $\mu$. The elements of $G$ are of the form $x=\left(x_{0}, x_{1}, \ldots, x_{k}, \ldots\right)$ with coordinates $x_{k} \in\{0,1\}(k \in \mathbb{N})$. The group operation on $G$ is the coordinate-wise addition modulo 2. A base for the neighborhoods of $G$ can be given in the following way:

$$
I_{0}(x):=G, \quad I_{n}(x):=\left\{y \in G: y=\left(x_{0}, \ldots, x_{n-1}, y_{n}, y_{n+1}, \ldots\right)\right\},
$$

$(x \in G, n \in \mathbb{N})$. These sets are called dyadic intervals containing $x$. Let $0=(0: i \in \mathbb{N}) \in G$ denote the null element of $G$. Let us set $I_{n}:=I_{n}(0)(n \in \mathbb{N})$ and $e_{n}:=(0, \ldots, 0,1,0, \ldots) \in$ $G$, where the $n$th coordinate is 1 and the rest are zeros $(n \in \mathbb{N})$. 
The $k$ th Rademacher function is given by

$$
r_{k}(x):=(-1)^{x_{k}} \quad(k \in \mathbb{N}, x \in G) .
$$

The Walsh-Paley system is defined as the product system of Rademacher functions. Namely, every natural number $n$ can be expressed in the number system of base 2 , in the form $n=\sum_{i=0}^{\infty} n_{i} 2^{i}$, where $n_{i} \in\{0,1\}$ is called the $i$ th coordinate of $n(i \in \mathbb{N})$.

Let us define the order $|n|$ of $n$ by $|n|:=\max \left\{j \in \mathbb{N}: n_{j} \neq 0\right\}$, that is $2^{|n|} \leq n<2^{|n|+1}$.

The sequence of Walsh-Paley functions is given by (for details see e.g. [10, 18])

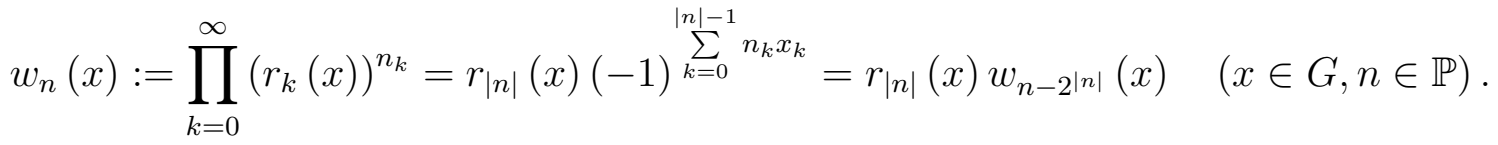

The Walsh-Kaczmarz functions are defined by $\kappa_{0}=1$ and for $n \geq 1$

$$
\kappa_{n}(x):=r_{|n|}(x) \prod_{k=0}^{|n|-1}\left(r_{|n|-1-k}(x)\right)^{n_{k}}=r_{|n|}(x)(-1)^{\sum_{k=0}^{|n|-1} n_{k} x_{|n|-1-k}}
$$

V. A. Skvortsov (see [24]) gave a relation between the Walsh-Kaczmarz functions and the Walsh-Paley functions by the help of a coordinate-transformation $\tau_{A}: G \rightarrow G$ given by

$$
\tau_{A}(x):=\left(x_{A-1}, x_{A-2}, \ldots, x_{1}, x_{0}, x_{A}, x_{A+1}, \ldots\right)
$$

for $A \in \mathbb{N}$. By the definition of $\tau_{A}$, we have

$$
\kappa_{n}(x)=r_{|n|}(x) w_{n-2|n|}\left(\tau_{|n|}(x)\right) \quad(n \in \mathbb{N}, x \in G) .
$$

The Dirichlet kernels and partial sums are defined by

$$
D_{n}^{\alpha}:=\sum_{k=0}^{n-1} \alpha_{k}, \quad S_{n}^{\alpha}(f ; x):=\sum_{k=0}^{n-1} \widehat{f}^{\alpha}(k) \alpha_{k}(x)
$$

for both system $\alpha_{n}=w_{n}(n \in \mathbb{N})$ and $\alpha_{n}=\kappa_{n}(n \in \mathbb{N})$, separately, Let us set $D_{0}^{\alpha}:=0$. The $2^{n}$-th Dirichlet kernels have a closed form (for details see e.g. [10, 18])

$$
D_{2^{n}}^{w}(x)=D_{2^{n}}^{\kappa}(x)=D_{2^{n}}(x)= \begin{cases}0, & \text { if } x \notin I_{n} \\ 2^{n}, & \text { if } x \in I_{n}\end{cases}
$$

The $n$th Fejér mean and kernel of the Walsh-(Kaczmarz)-Fourier series of a function $f$ is given by

$$
\sigma_{n}^{\alpha}(f ; x)=\frac{1}{n} \sum_{j=0}^{n} S_{j}^{\alpha}(f ; x), \quad K_{n}^{\alpha}(x):=\frac{1}{n} \sum_{k=0}^{n} D_{k}^{\alpha}(x) .
$$

It is known that (for details see e.g. [10, 18]) there exists a positive constant $C$ such that

$$
\left\|K_{n}^{\kappa}\right\|_{1} \leq C \text { for all } n \in \mathbb{N} \text {. }
$$




\section{HARDY SPACES AND AUXILIARY PROPOSITIONS}

To prove our main Theorem we need the following Lemmas and definitions in [7, 24, 33, 34].

Lemma 2.1 (Skvortsov [24]). For $n \in \mathbb{P}, x \in G$

$$
\begin{aligned}
n K_{n}^{\kappa}(x) & =1+\sum_{i=0}^{|n|-1} 2^{i} D_{2^{i}}(x)+\sum_{i=0}^{|n|-1} 2^{i} r_{i}(x) K_{2^{i}}^{w}\left(\tau_{i}(x)\right) \\
& +\left(n-2^{|n|}\right)\left(D_{2^{|n|}}(x)+r_{|n|}(x) K_{n-2^{|n|}}^{w}\left(\tau_{|n|}(x)\right)\right) .
\end{aligned}
$$

Lemma 2.2 (Gát [5]). Let $A, t \in \mathbb{N}, A>t$. Suppose that $x \in I_{t} \backslash I_{t+1}$. Then

$$
K_{2^{A}}^{\omega}(x)= \begin{cases}0, & \text { if } x-x_{t} e_{t} \notin I_{A} \\ 2^{t-1}, & \text { if } x-x_{t} e_{t} \in I_{A}\end{cases}
$$

If $x \in I_{A}$, then $K_{2^{A}}^{\omega}(x)=\frac{2^{A}+1}{2}$.

Lemma 2.3 (Gát, Goginava, Nagy [7]). Let $n<2^{A+1}, A>N$ and $x \in I_{N}\left(x_{0}, \ldots, x_{m-1}, x_{m}=\right.$ $\left.1,0, \ldots, 0, x_{l}=1,0, \ldots, 0\right)=: J_{N}^{m, l}, l=0, \ldots, N-1, m=-1,0, \ldots, l$. Then

$$
\int_{I_{N}} n\left|K_{n}^{w}\left(\tau_{A}(x+t)\right)\right| d t \leq c \frac{2^{A}}{2^{m+l}}
$$

where

$$
I_{N}\left(x_{0}, \ldots, x_{m}=1,0, \ldots, 0, x_{l}=1,0, \ldots, 0\right):=I_{N}\left(0, \ldots, 0, x_{l}=1,0, \ldots, 0\right)
$$

for $m=-1$.

The $\sigma$-algebra generated by the dyadic intervals of measure $2^{-k}$ will be denoted by $\mathcal{F}_{k}$ $(k \in \mathbb{P})$. Denote by $f=\left(f^{(n)}, n \in \mathbb{P}\right)$ a martingale with respect to $\left(\mathcal{F}_{n}, n \in \mathbb{P}\right)$ (for details see, e. g. [34]). The maximal function of a martingale $f$ is defined by

$$
f^{*}=\sup _{n \in \mathbb{P}}\left|f^{(n)}\right| \text {. }
$$

In case $f \in L_{1}(G)$, the maximal function can also be given by

$$
f^{*}(x)=\sup _{n \in \mathbb{P}} \frac{1}{\mu\left(I_{n}(x)\right)}\left|\int_{I_{n}(x)} f(u) d \mu(u)\right|, \quad x \in G .
$$

For $0<p<\infty$ the Hardy martingale space $H_{p}(G)$ consists of all martingales for which

$$
\|f\|_{H_{p}}:=\left\|f^{*}\right\|_{p}<\infty .
$$

If $f \in L_{1}(G)$, then it is easy to show that the sequence $\left(S_{2^{n}} f: n \in \mathbb{P}\right)$ is a martingale. If $f$ is a martingale, that is $f=\left(f^{(0)}, f^{(1)}, \ldots\right)$ then the Walsh-(Kaczmarz)-Fourier coefficients must be defined in a little bit different way:

$$
\widehat{f}(i)=\lim _{k \rightarrow \infty} \int_{G} f^{(k)}(x) \alpha_{i}(x) d \mu(x), \quad\left(\alpha_{i}=w_{i} \text { or } \kappa_{i}\right) .
$$


The Walsh-(Kaczmarz)-Fourier coefficients of $f \in L_{1}(G)$ are the same as the ones of the martingale $\left(S_{2^{n}} f: n \in \mathbb{P}\right)$ obtained from $f$.

A useful characterization of the Hardy spaces $H_{p}$ is the atomic structure. A bounded measurable function $a$ is a $p$-atom, if there exists a dyadic interval $I$, such that

a) $\int_{I} a d \mu=0$

b) $\|a\|_{\infty} \leq \mu(I)^{-1 / p}$,

c) $\operatorname{supp} a \subset I$.

Hardy martingale spaces $H_{p}(G)$ for $0<p \leq 1$ have atomic characterizations (see e.g. Weisz [33, 34]):

Lemma 2.4 (Weisz [33]). A martingale $f=\left(f^{(n)}: n \in \mathbb{N}\right)$ is in $H_{p}(0<p \leq 1)$ if and only if there exist a sequence $\left(a_{k}, k \in \mathbb{N}\right)$ of $p$-atoms and a sequence $\left(\mu_{k}: k \in \mathbb{N}\right)$ of real numbers such that, for every $n \in \mathbb{N}$,

$$
\sum_{k=0}^{\infty} \mu_{k} S_{2^{n}}\left(a_{k}\right)=f^{(n)}, \quad \text { a.e. }
$$

where

$$
\sum_{k=0}^{\infty}\left|\mu_{k}\right|^{p}<\infty
$$

Moreover,

$$
\|f\|_{H_{p}} \backsim \inf \left(\sum_{k=0}^{\infty}\left|\mu_{k}\right|^{p}\right)^{1 / p},
$$

where the infimum is taken over all decomposition of $f=\left(f^{(n)}: n \in \mathbb{N}\right)$ of the form (2.1).

Lemma 2.5 (Weisz [34]). Suppose that the operator $T$ is $\sigma$-sublinear and for some $0<p \leq 1$

$$
\int_{\bar{I}}|T a|^{p} d \mu \leq c_{p}<\infty
$$

for every p-atom a, where I denotes the support of the atom. If $T$ is bounded from $L_{\infty}$ to $L_{\infty}$, then

$$
\|T f\|_{p} \leq c_{p}\|f\|_{H_{p}} \quad \text { for all } f \in H_{p}
$$

For a martingale

$$
f=\sum_{n=0}^{\infty}\left(f_{n}-f_{n-1}\right)
$$

the conjugate transforms are defined as

$$
\widetilde{f^{(t)}}=\sum_{n=0}^{\infty} r_{n}(t)\left(f_{n}-f_{n-1}\right),
$$

where $t \in G$ is fixed. Note that $\widetilde{f^{(0)}}=f$. It is well-known (see [33]) that

$$
\begin{aligned}
\| \widetilde{f^{(t)} \|_{H_{p}(G)}} & =\|f\|_{H_{p}(G)},\|f\|_{H_{p}(G)}^{p} \sim \int_{G}\left\|\widetilde{f^{(t)}}\right\|_{p}^{p} d \mu(t), \\
\left(\widetilde{\left.\sigma_{m}^{\kappa}(f)\right)^{(t)}}\right. & =\sigma_{m}^{\kappa}\left(\widetilde{(f)^{(t)}}\right) .
\end{aligned}
$$




\section{Strong CONVERGEnCE THEOREM And CONNECTING RESUltS}

Our main Theorem reads as follows.

Theorem 3.1. Let $0<p \leq 1 / 2$. Then there exists a positive constant $c_{p}$ depending only on $p$, such that

$$
\frac{1}{\log ^{[p+1 / 2]} n} \sum_{m=1}^{n} \frac{\left\|\sigma_{m}^{\kappa}(f)\right\|_{H_{p}}^{p}}{m^{2-2 p}} \leq c_{p}\|f\|_{H_{p}}^{p}
$$

holds for all $f \in H_{p}$.

Proof. During the proof of our main theorem we use the notation and some basic result of paper [9]. Let us suppose that

$$
\frac{1}{\log ^{[p+1 / 2]} n} \sum_{m=1}^{n} \frac{\left\|\sigma_{m}^{\kappa}(f)\right\|_{p}^{p}}{m^{2-2 p}} \leq c_{p}\|f\|_{H_{p}}^{p}
$$

holds for all $f \in H_{p}$. Combining (2.2) and (3.1) we have that

$$
\begin{aligned}
& \frac{1}{\log ^{[p+1 / 2]} n} \sum_{m=1}^{n} \frac{\left\|\sigma_{m}^{\kappa}(f)\right\|_{H_{p}}^{p}}{m^{2-2 p}} \sim \frac{1}{\log ^{[p+1 / 2]} n} \sum_{m=1}^{n} \int_{G} \frac{\left\|\widetilde{\left(\sigma_{m}^{\kappa}(f)\right)^{(t)}}\right\|_{p}^{p}}{m^{2-2 p}} d \mu(t) \\
= & \int_{G} \frac{1}{\log ^{[p+1 / 2]} n} \sum_{m=1}^{n} \frac{\widehat{\left(\sigma_{m}^{\kappa}(f)\right)^{(t)}} \|_{p}^{p}}{m^{2-2 p}} d \mu(t)=\int_{G} \frac{1}{\log ^{[p+1 / 2]} n} \sum_{m=1}^{n} \frac{\| \sigma_{m}^{\kappa}\left(\widetilde{\left.(f)^{(t)}\right)} \|_{p}^{p}\right.}{m^{2-2 p}} d \mu(t) \\
\leq & c_{p} \int_{G}\left\|\widetilde{(f)^{(t)}}\right\|_{H_{p}}^{p} d \mu(t) \sim c_{p} \int_{G}\|f\|_{H_{p}}^{p} d \mu(t) \sim c_{p}\|f\|_{H_{p}}^{p} .
\end{aligned}
$$

Since $\sigma_{n}^{\kappa}$ are bounded (see inequality (1.4) ) from the space $L_{\infty}$ to the space $L_{\infty}$, by Lemma 2.5 it is enough to prove that

$$
\frac{1}{\log { }^{[p+1 / 2]} n} \sum_{m=1}^{n} \frac{\left\|\sigma_{m}^{\kappa}(a)\right\|_{p}^{p}}{m^{2-2 p}}<c_{p}<\infty
$$

for every arbitrary $p$-atom $a$. This leads us to inequality (3.1).

Let $a$ be an arbitrary $p$-atom with support $I$ and $\mu(I)=2^{-N}$. Without loss of generality, we may assume that $I:=I_{N}$. It is easily seen that $\sigma_{n}^{\kappa}(a)=0$ if $n \leq 2^{N}$. Therefore, we set $n>2^{N}$.

We can write

$$
\begin{aligned}
\frac{1}{\log ^{[p+1 / 2]} n} & \sum_{m=1}^{n} \frac{\left\|\sigma_{m}^{\kappa}(a)\right\|_{p}^{p}}{m^{2-2 p}} \leq \frac{1}{\log ^{[p+1 / 2]} n} \sum_{m=2^{N}}^{n} \frac{\left\|\sigma_{m}^{\kappa}(a)\right\|_{p}^{p}}{m^{2-2 p}} \\
& \leq \frac{1}{\log ^{[p+1 / 2]} n} \sum_{m=2^{N}}^{n} \int_{I_{N}} \frac{\left|\sigma_{m}^{\kappa}(a)\right|^{p}}{m^{2-2 p}} d \mu+\frac{1}{\log ^{[p+1 / 2]} n} \sum_{m=2^{N}}^{n} \int_{\overline{I_{N}}} \frac{\left|\sigma_{m}^{\kappa}(a)\right|^{p}}{m^{2-2 p}} d \mu \\
& =: I_{1}+I_{2} .
\end{aligned}
$$


Inequality (1.4) implies

$$
\begin{aligned}
I_{1} & \leq \frac{1}{\log [p+1 / 2]} n \sum_{m=2^{N}}^{\infty} \int_{I_{N}} \frac{\left|\sigma_{m}^{\kappa}(a)\right|^{p}}{m^{2-2 p}} d \mu \\
& \leq \frac{c_{p}}{\log [p+1 / 2]} n \sum_{m=2^{N}}^{\infty} \frac{1}{m^{2-2 p}}\|a\|_{\infty}^{p} / 2^{N} \\
& \leq \frac{c_{p}}{\log [p+1 / 2]} n \sum_{m=2^{N}}^{n} \frac{1}{m^{2-2 p}}<c_{p}
\end{aligned}
$$

for $0<p \leq 1 / 2$.

Now, we estimate the expression $I_{2}$. Lemma 2.1 yields that

$$
\begin{aligned}
\left|\sigma_{n}^{\kappa}(f)\right| & =\left|f * K_{n}^{\kappa}\right| \leq\left|f * \frac{1}{n}\left(1+\sum_{i=0}^{|n|-1} 2^{i} D_{2^{i}}\right)\right| \\
& +\left|f * \frac{1}{n} \sum_{i=0}^{|n|-1} 2^{i} r_{i} K_{2^{i}}^{w} \circ \tau_{i}\right|+\left|f * \frac{n-2^{|n|}}{n}\left(D_{2^{|n|}}+r_{|n|} K_{n-2}^{w} \circ \tau_{|n|}\right)\right| \\
& =: \sum_{i=1}^{3}\left|f * L_{n}^{i}\right| .
\end{aligned}
$$

It is easily seen that

$$
\begin{aligned}
\left|\left(a * L_{n}^{i}\right)(x)\right| & \leq \int_{I_{N}}|a(s)|\left|L_{n}^{i}(x+s)\right| d \mu(s) \leq\|a\|_{\infty} \int_{I_{N}}\left|L_{n}^{i}(x+s)\right| d \mu(s) \\
& \leq 2^{N / p} \int_{I_{N}}\left|L_{n}^{i}(x+s)\right| d \mu(s)
\end{aligned}
$$

for $i=1,2,3\left(\right.$ and $\left.n>2^{N}\right)$. For expression $I_{2}$ we have

$$
I_{2} \leq \frac{1}{\log { }^{[p+1 / 2]} n} \sum_{m=2^{N}}^{n} \frac{\sum_{i=1}^{3} \int \frac{\int_{I}}{I_{N}}\left|\left(a * L_{m}^{i}\right)(x)\right|^{p} d \mu(x)}{m^{2-2 p}}=: I_{2}^{1}+I_{2}^{2}+I_{2}^{3} .
$$

First, we discuss the expression $I_{2}^{1}$. We decompose the set $\overline{I_{N}}$ as

$$
\overline{I_{N}}=\bigcup_{j=0}^{N-1}\left(I_{j} \backslash I_{j+1}\right) .
$$

Set $x \in I_{j} \backslash I_{j+1}$ and $s \in I_{N}$, then $x+s \in I_{j} \backslash I_{j+1}$ for $j=0, \ldots, N-1$. Applying (3.3) and (1.3), we have

$$
\begin{aligned}
\int_{I_{N}}\left|L_{m}^{1}(x+s)\right| d \mu(s) & \leq \int_{I_{N}} \frac{1}{m}\left(1+\sum_{i=0}^{j} 2^{i} D_{2^{i}}(x+s)\right) d \mu(s) \\
& \leq \frac{c}{m} 2^{2 j} 2^{-N}
\end{aligned}
$$


and

$$
\begin{aligned}
I_{2}^{1} & \leq \frac{1}{\log { }^{[p+1 / 2]} n} \sum_{m=2^{N}}^{n} \frac{\sum_{t=0}^{N-1} \int_{I_{t} \backslash I_{t+1}}\left|\left(a * L_{m}^{1}\right)(x)\right|^{p} d \mu(x)}{m^{2-2 p}} \\
& \leq \frac{1}{\log { }^{[p+1 / 2]} n} \sum_{m=2^{N}}^{n} \frac{\sum_{t=0}^{N-1} \frac{2^{N} 2^{2 t p}}{m^{p} 2^{N p}} 2^{-t}}{m^{2-2 p}} \\
& \leq \frac{c}{\log ^{[p+1 / 2]} n} \sum_{m=2^{N}}^{n} \frac{2^{N(1-p)}}{m^{2-p}} \sum_{t=0}^{N-1} 2^{t(2 p-1)} .
\end{aligned}
$$

If $p=1 / 2$ we have

$$
I_{2}^{1} \leq \frac{c}{\log n} \sum_{m=2^{N}}^{n} \frac{2^{N / 2}}{m^{3 / 2}} N \leq c
$$

and if $0<p<1 / 2$

$$
I_{2}^{1} \leq c_{p} \sum_{m=2^{N}}^{n} \frac{2^{N(1-p)}}{m^{2-p}} \leq c_{p}
$$

Second, we discuss the expression $I_{2}^{2}$. We use the disjoint decomposition (3.4) of $\overline{I_{N}}$ and we decompose the sets $I_{t} \backslash I_{t+1}$ as the following disjoint union:

$$
I_{t} \backslash I_{t+1}=\bigcup_{l=t+1}^{N} J_{t}^{l},
$$

where $J_{t}^{l}:=I_{N}\left(0, \ldots, 0, x_{t}=1,0, \ldots, 0, x_{l}=1, x_{l+1}, \ldots, x_{N-1}\right)$ for $t<l<N$ and $J_{t}^{l}:=I_{N}\left(e_{t}\right)$ for $l=N$. Let $x \in J_{t}^{l}$ and $s \in I_{N}$, then $x+s \in J_{t}^{l}(0 \leq t<N, t<l \leq N)$.

For $0 \leq t<l<N$, the next inequality showed in [9, page 681.]

$$
\int_{I_{N}}\left|L_{m}^{2}(x+s)\right| d \mu(s) \leq c \frac{2^{2 t}+2^{2 l-t}}{m} 2^{-N} .
$$

For $0 \leq t<l=N$, it is showed in [9, page 681.] that

$$
\int_{I_{N}}\left|L_{m}^{2}(x+s)\right| d \mu(s) \leq c \frac{2^{2 t-N}+2^{N-t}+2^{|m|-t}}{m} .
$$

The decomposition of $\overline{I_{N}}$ yields

$$
\begin{aligned}
\int_{\overline{I_{N}}}\left|\left(a * L_{m}^{2}\right)(x)\right|^{p} d \mu(x)= & \sum_{t=0}^{N-1} \int_{I_{t} \backslash I_{t+1}}\left|\left(a * L_{m}^{2}\right)(x)\right|^{p} d \mu(x) \\
= & \sum_{t=0}^{N-1} \sum_{l=t+1}^{N-1} \int_{J_{t}^{l}}\left|\left(a * L_{m}^{2}\right)(x)\right|^{p} d \mu(x) \\
& +\sum_{t=0}^{N-1} \int_{J_{t}^{N}}\left|\left(a * L_{m}^{2}\right)(x)\right|^{p} d \mu(x)
\end{aligned}
$$


and

$$
\begin{aligned}
I_{2}^{2} \leq & \frac{1}{\log ^{[p+1 / 2]} n} \sum_{m=2^{N}}^{n} \frac{\sum_{t=0}^{N-1} \sum_{l=t+1}^{N-1} \int_{J_{t}^{l}}\left|\left(a * L_{m}^{2}\right)(x)\right|^{p} d \mu(x)}{m^{2-2 p}} \\
& +\frac{1}{\log ^{[p+1 / 2]} n} \sum_{m=2^{N}}^{n} \frac{\sum_{t=0}^{N-1} \int_{J_{t}^{N}}\left|\left(a * L_{m}^{2}\right)(x)\right|^{p} d \mu(x)}{m^{2-2 p}} \\
=: & I_{2}^{2,1}+I_{2}^{2,2} .
\end{aligned}
$$

For $I_{2}^{2,1}$ we apply inequality (3.3) and (3.5)

$$
\begin{aligned}
I_{2}^{2,1} & \leq \frac{1}{\log ^{[p+1 / 2]} n} \sum_{m=2^{N}}^{n} \frac{\sum_{t=0}^{N-1} \sum_{l=t+1}^{N-1} c_{p} 2^{N} \frac{\left(2^{2 t}+2^{2 l-t}\right)^{p}}{m^{p}} 2^{-N p} 2^{-l}}{m^{2-2 p}} \\
& \leq \frac{c_{p}}{\log ^{[p+1 / 2]} n} \sum_{m=2^{N}}^{n} 2^{N(1-p)} \frac{\sum_{t=0}^{N-1} \sum_{l=t+1}^{N-1}\left(2^{2 t p}+2^{(2 l-t) p}\right) 2^{-l}}{m^{2-p}} .
\end{aligned}
$$

For $p=1 / 2$ we get

$$
I_{2}^{2,1} \leq \frac{c_{p}}{\log n} 2^{N / 2} \sum_{m=2^{N}}^{n} \frac{N}{m^{3 / 2}} \leq c_{p}
$$

and for $0<p<1 / 2$ we have

$$
I_{2}^{2,1} \leq c_{p} 2^{N(1-p)} \sum_{m=2^{N}}^{n} \sum_{t=0}^{N-1} \sum_{l=t+1}^{N-1} \frac{2^{2 t p-l}+2^{l(2 p-1)-t p}}{m^{2-p}} \leq 2^{N(1-p)} \sum_{m=2^{N}}^{n} \frac{c_{p}}{m^{2-p}} \leq c_{p} .
$$

By inequality (3.3) and (3.6) we write

$$
\begin{aligned}
I_{2}^{2,2} & \leq \frac{c_{p}}{\log ^{[p+1 / 2]} n} \sum_{m=2^{N}}^{n} \frac{\sum_{t=0}^{N-1} 2^{N} \frac{\left(2^{2 t-N}+2^{N-t}+2^{|m|-t}\right)^{p}}{m^{p}} 2^{-N}}{m^{2-2 p}} \\
& \leq \frac{c_{p}}{\log ^{[p+1 / 2]} n} \sum_{m=2^{N}}^{n} \frac{\sum_{t=0}^{N-1}\left(2^{(2 t-N) p}+2^{(|m|-t) p}\right)}{m^{2-p}} .
\end{aligned}
$$

We devide the expression $I_{2}^{2,2}$ into two parts

$$
\begin{aligned}
\frac{c_{p}}{\log ^{[p+1 / 2]} n} \sum_{m=2^{N}}^{n} \frac{1}{m^{2-p}} \sum_{t=0}^{N-1} 2^{(2 t-N) p} & \leq \frac{c_{p}}{\log ^{[p+1 / 2]} n} \sum_{m=2^{N}}^{n} \frac{1}{m^{2-p}} 2^{N p} \\
& \leq \frac{c_{p}}{\log ^{[p+1 / 2]} n} 2^{N(2 p-1)} \leq c_{p}
\end{aligned}
$$

and

$$
\frac{c_{p}}{\log ^{[p+1 / 2]} n} \sum_{m=2^{N}}^{n} \frac{1}{m^{2-2 p}} \sum_{t=0}^{N-1} \frac{2^{(|m|-t) p}}{m^{p}} \leq \frac{c_{p}}{\log ^{[p+1 / 2]} n} \sum_{m=2^{N}}^{n} \frac{1}{m^{2-2 p}} \leq c_{p}
$$

for $0<p \leq 1 / 2$.

At last, we discuss the expression $I_{2}^{3}$. We use Lemma 2.3 and the following disjoint decomposition of $\overline{I_{N}}$ :

$$
\overline{I_{N}}=\bigcup_{l=0}^{N-1} \bigcup_{k=-1}^{l} J_{N}^{k, l}
$$


where the set $J_{N}^{k, l}$ is defined in Lemma 2.3.

If $x \in \overline{I_{N}}$ and $s \in I_{N}$, then $x+s \in \overline{I_{N}}$ and $D_{2^{|n|}}(x+s)=0$. Moreover, if $x \in J_{N}^{k, l}$, then $x+s \in J_{N}^{k, l}$ and by Lemma 2.3 we have

$$
\int_{I_{N}}\left|L_{m}^{3}(x+s)\right| d \mu(s) \leq c \frac{2^{|m|}}{m 2^{l+k}} .
$$

The decomposition of $\overline{I_{N}}$ yields

$$
\int_{\overline{I_{N}}}\left|\left(a * L_{m}^{3}\right)(x)\right|^{p} d \mu(x)=\sum_{l=0}^{N-1} \sum_{k=-1}^{l} \int_{J_{N}^{k, l}}\left|\left(a * L_{m}^{3}\right)(x)\right|^{p} d \mu(x) .
$$

Inequalities (3.3), (3.8) and (3.9) yield

$$
\begin{aligned}
I_{2}^{3} & \leq \frac{1}{\log ^{[p+1 / 2]} n} \sum_{m=2^{N}}^{n} \frac{\sum_{l=0}^{N-1} \sum_{k=-1}^{l} \int_{J_{N}^{k, k}}\left|\left(a * L_{m}^{3}\right)(x)\right|^{p} d \mu(x)}{m^{2-2 p}} \\
& \leq \frac{c_{p}}{\log ^{[p+1 / 2]} n} \sum_{m=2^{N}}^{n} \frac{\sum_{l=0}^{N-1} \sum_{k=-1}^{l} 2^{-N} 2^{(-l-k) p} 2^{-(N-k)}}{m^{2-2 p}} \\
& \leq \frac{c_{p}}{\log ^{[p+1 / 2]} n} \sum_{m=2^{N}}^{n} 2^{-2 N} \frac{\sum_{l=0}^{N-1} 2^{-l p} \sum_{k=-1}^{l} 2^{k(1-p)}}{m^{2-2 p}} \\
& \leq \frac{c_{p}}{\log ^{[p+1 / 2]} n} \sum_{m=2^{N}}^{n} 2^{-2 N} \frac{\sum_{l=0}^{N-1} 2^{l(1-2 p)}}{m^{2-2 p}} \leq c_{p}
\end{aligned}
$$

for $0<p \leq 1 / 2$. Summarizing our results for expressions $I_{1}, I_{2}^{1}, I_{2}^{2,1}, I_{2}^{2,2}$ and $I_{2}^{3}$ we complete the proof of our main Theorem 3.1 .

In the next theorem we show the sharpness of the statement of Theorem 3.1 in case $0<$ $p<1 / 2$.

Theorem 3.2. Let $0<p<1 / 2$ and $\Phi: \mathbb{N}_{+} \rightarrow[1, \infty)$ is any non-decreasing function, satisfying the conditions $\Phi(n) \uparrow \infty$ and

$$
\varlimsup_{k \rightarrow \infty} \frac{2^{k(2-2 p)}}{\Phi\left(2^{k}\right)}=\infty .
$$

Then there exists a martingale $F \in H_{p}$, such that

$$
\sum_{m=1}^{\infty} \frac{\left\|\sigma_{m} F\right\|_{L_{p, \infty}}^{p}}{\Phi(m)}=\infty
$$

Proof. Let $\Phi(n)$ be non-decreasing function, satisfying condition

$$
\lim _{k \rightarrow \infty} \frac{2^{\left(\left|n_{k}\right|+1\right)(2-2 p)}}{\Phi\left(2^{\left|n_{k}\right|+1}\right)}=\infty .
$$

Under condition (3.10), there exists a sequence $\left\{\alpha_{k}: k \geq 0\right\} \subset\left\{n_{k}: k \geq 0\right\}$ such that

$$
\left|\alpha_{k}\right| \geq 2, \quad \text { for all } k \geq 0
$$


and

$$
\sum_{\eta=0}^{\infty} \frac{\Phi^{1 / 2}\left(2^{\left|\alpha_{\eta}\right|+1}\right)}{2^{\left|\alpha_{\eta}\right|(1-p)}}=2^{1-p} \sum_{\eta=0}^{\infty} \frac{\Phi^{1 / 2}\left(2^{\left|\alpha_{\eta}\right|+1}\right)}{2^{\left(\left|\alpha_{\eta}\right|+1\right)(1-p)}}<c<\infty
$$

Let

$$
F_{n}=\sum_{\left\{k:\left|\alpha_{k}\right|<n\right\}} \lambda_{k} a_{k}
$$

where

$$
\lambda_{k}=\frac{\Phi^{1 / 2 p}\left(2^{\left|\alpha_{k}\right|+1}\right)}{2^{\left(\left|\alpha_{k}\right|\right)(1 / p-1)}}
$$

and

$$
a_{k}=2^{\left|\alpha_{k}\right|(1 / p-1)}\left(D_{2^{\left|\alpha_{k}\right|+1}}-D_{2^{\mid \alpha_{k}} \mid}\right) .
$$

It is easy to show that the martingale $F=\left(F_{n}, n \in \mathbb{N}\right) \in H_{p}$. Indeed, since

$$
\begin{gathered}
S_{2^{n}} a_{k}= \begin{cases}a_{k}, & \left|\alpha_{k}\right|<n, \\
0, & \left|\alpha_{k}\right| \geq n,\end{cases} \\
\operatorname{supp}\left(a_{k}\right)=I_{\left|\alpha_{k}\right|}, \quad \int_{I_{\left|\alpha_{k}\right|}} a_{k} d \mu=0 \quad \text { and } \quad\left\|a_{k}\right\|_{\infty} \leq 2^{\left|\alpha_{k}\right| / p}=\left(\mu\left(\operatorname{supp} a_{k}\right)\right)^{-1 / p}
\end{gathered}
$$

if we apply Lemma 2.4 and (3.12) we conclude that $F \in H_{p}$.

It is easily seen that

$$
\widehat{F}^{\kappa}(j)= \begin{cases}\Phi^{1 / 2 p}\left(2^{\left|\alpha_{k}\right|+1}\right), & \text { if } j \in\left\{2^{\left|\alpha_{k}\right|}, \ldots, 2^{\left|\alpha_{k}\right|+1}-1\right\}, k=0,1,2 \ldots \\ 0, & \text { if } j \notin \bigcup_{k=0}^{\infty}\left\{2^{\left|\alpha_{k}\right|}, \ldots, 2^{\left|\alpha_{k}\right|+1}-1\right\}\end{cases}
$$

Let $2^{\left|\alpha_{k}\right|}<n<2^{\left|\alpha_{k}\right|+1}$. Using (3.13) we can write

$$
\sigma_{n}^{\kappa} F=\frac{1}{n} \sum_{j=1}^{2^{\left|\alpha_{k}\right|}} S_{j}^{\kappa} F+\frac{1}{n} \sum_{j=2^{\left|\alpha_{k}\right|}+1}^{n} S_{j}^{\kappa} F=I I I+I V .
$$

It is simple to show that

$$
S_{j}^{\kappa} F=\left\{\begin{array}{l}
0, \quad \text { if } 0 \leq j \leq 2^{\left|\alpha_{0}\right|} \\
\Phi^{1 / 2 p}\left(2^{\left|\alpha_{0}\right|+1}\right)\left(D_{j}^{\kappa}-D_{2^{\left|\alpha_{0}\right|}}\right), \quad \text { if } \quad 2^{\left|\alpha_{0}\right|}<j \leq 2^{\left|\alpha_{0}\right|+1} .
\end{array}\right.
$$


Suppose that $2^{\left|\alpha_{s}\right|}<j \leq 2^{\left|\alpha_{s}\right|+1}$, for some $s=1,2, \ldots, k$. Then applying (3.13) we have

$$
\begin{aligned}
S_{j}^{\kappa} F= & \sum_{v=0}^{2^{\left|\alpha_{s-1}\right|+1}-1} \widehat{F}^{\kappa}(v) \kappa_{v}+\sum_{v=2^{\left|\alpha_{s}\right|}}^{j-1} \widehat{F}^{\kappa}(v) \kappa_{v} \\
= & \sum_{\eta=0}^{s-1} \sum_{v=2^{\left|\alpha_{\eta}\right|}}^{2^{\left|\alpha_{\eta}\right|+1}-1} \widehat{F}^{\kappa}(v) \kappa_{v}+\sum_{v=2^{\left|\alpha_{s}\right|}}^{j-1} \widehat{F}^{\kappa}(v) \kappa_{v} \\
= & \sum_{\eta=0}^{s-1} \sum_{v=2}^{2^{\left|\alpha_{\eta}\right|+1}-1} \Phi^{1 / 2 p}\left(2^{\left|\alpha_{\eta}\right|+1}\right) \kappa_{v}+\Phi^{1 / 2 p}\left(2^{\left|\alpha_{s}\right|+1}\right) \sum_{v=2^{\left|\alpha_{s}\right|}}^{j-1} \kappa_{v} \\
= & \sum_{\eta=0}^{s-1} \Phi^{1 / 2 p}\left(2^{\left|\alpha_{\eta}\right|+1}\right)\left(D_{2^{\left|\alpha_{\eta}\right|+1}}-D_{2^{\left|\alpha_{\eta}\right|}}\right) \\
& +\Phi^{1 / 2 p}\left(2^{\left|\alpha_{s}\right|+1}\right)\left(D_{j}^{\kappa}-D_{2^{\left|\alpha_{s}\right|}}\right) .
\end{aligned}
$$

Let $2^{\left|\alpha_{s}\right|+1} \leq j \leq 2^{\left|\alpha_{s+1}\right|}, s=0,1, \ldots k-1$. Analogously of (3.16) we get

$$
S_{j}^{\kappa} F=\sum_{v=0}^{2^{\left|\alpha_{s}\right|+1}} \widehat{F}^{\kappa}(v) \kappa_{v}=\sum_{\eta=0}^{s} \Phi^{1 / 2 p}\left(2^{\left|\alpha_{\eta}\right|+1}\right)\left(D_{2^{\left|\alpha_{\eta}\right|+1}}-D_{2^{\left|\alpha_{\eta}\right|}}\right) .
$$

Let $x \in I_{2}\left(e_{0}+e_{1}\right)$. Since (see (1.3) $)$ Lemma 2.1 Lemma 2.2)

$$
D_{2^{n}}(x)=K_{2^{n}}^{\kappa}(x)=K_{2^{n+1}}^{\kappa}(x)-K_{2^{n}}^{\kappa}(x)=0, \text { for } n \geq 2,
$$

from (3.11) and (3.16+3.17) we obtain

$$
\begin{gathered}
I I I=\frac{1}{n} \sum_{\eta=0}^{k-1} \Phi^{1 / 2 p}\left(2^{\left|\alpha_{\eta}\right|+1}\right) \sum_{v=2^{\left|\alpha_{\eta}\right|+1}}^{2^{\left|\alpha_{\eta}\right|+1}} D_{v}^{\kappa}(x) \\
=\frac{1}{n} \sum_{\eta=0}^{k-1} \Phi^{1 / 2 p}\left(2^{\left|\alpha_{\eta}\right|+1}\right)\left(2^{\left|\alpha_{\eta}\right|+1} K_{2^{\left|\alpha_{\eta}\right|+1}}^{\kappa}(x)-2^{\left|\alpha_{\eta}\right|} K_{2^{\left|\alpha_{\eta}\right|}}^{\kappa}(x)\right)=0 .
\end{gathered}
$$

Applying (3.16), when $s=k$ in $I V$ we have

$$
\begin{aligned}
I V= & \frac{n-2^{\left|\alpha_{k}\right|}}{n} \sum_{\eta=0}^{k-1} \Phi^{1 / 2 p}\left(2^{\left|\alpha_{\eta}\right|+1}\right)\left(D_{2^{\left|\alpha_{\eta}\right|+1}}-D_{2^{\left|\alpha_{\eta}\right|}}\right) \\
& +\frac{\Phi^{1 / 2 p}\left(2^{\left|\alpha_{k}\right|+1}\right)}{n} \sum_{j=2^{\left|\alpha_{k}\right|+1}}^{n}\left(D_{j}^{\kappa}-D_{2^{\left|\alpha_{k}\right|}}\right)=I V_{1}+I V_{2} .
\end{aligned}
$$

Combining (3.11) and (3.18) we get

$$
I V_{1}=0, \text { for } x \in I_{2}\left(e_{0}+e_{1}\right) .
$$

Let $x \in I_{2}\left(e_{0}+e_{1}\right), 2^{\left|\alpha_{k}\right|}<n<2^{\left|\alpha_{k}\right|+1}$ and $n \in \mathbb{A}_{0,2}$, where $\mathbb{A}_{0,2}$ is defined by

$$
\mathbb{A}_{0,2}:=\left\{n \in \mathbb{N}: n=2^{0}+2^{2}+\sum_{i=3}^{s} n_{i} 2^{i}\right\} \text {. }
$$


We have that

$$
D_{j+2}^{\kappa}\left|\alpha_{k}\right|=\left.D_{2}\right|^{\left|\alpha_{k}\right|}+\sum_{j=2^{\left|\alpha_{k}\right|}}^{2^{\left|\alpha_{k}\right|}} \kappa_{j} \text {, when } j<2^{\left|\alpha_{k}\right|}
$$

from (3.18) and (3.22) we obtain

$$
\begin{aligned}
\left|I V_{2}\right| & =\frac{\Phi^{1 / 2 p}\left(2^{\left|\alpha_{k}\right|+1}\right)}{n}\left|\sum_{j=1}^{n-2^{\left|\alpha_{k}\right|}}\left(\left.D_{j+2}^{\kappa}\right|_{\alpha_{k} \mid}(x)-D_{2^{\left|\alpha_{k}\right|}}^{\kappa}(x)\right)\right| \\
& =\frac{\Phi^{1 / 2 p}\left(2^{\left|\alpha_{k}\right|+1}\right)}{n}\left|\sum_{j=1}^{n-2} \sum_{l=2^{\left|\alpha_{k}\right|}}^{\left|\alpha_{k}\right|} \kappa_{2_{l}\left|\alpha_{k}\right|+j-1}\right| \\
& =\frac{\Phi^{1 / 2 p}\left(2^{\left|\alpha_{k}\right|+1}\right)}{n}\left|\sum_{j=1}^{n-2} \sum_{i=0}^{\left|\alpha_{k}\right|} \kappa_{2^{\left|\alpha_{k}\right|+i}}(x)\right|
\end{aligned}
$$

It is obvious that every $n \in \mathbb{A}_{0,2}, 2^{\left|\alpha_{k}\right|}<n<2^{\left|\alpha_{k}\right|+1}$ can be expressed as $n=2^{\left|\alpha_{k}\right|}+4 k+1$ and quantity of the members of final double sums are odd numbers. Indeed, quantity of the members can be calculated by the following sum:

$$
\sum_{j=1}^{n-2} j=\left(n-2^{\left|\alpha_{k}\right|}\right)\left(n-2^{\left|\alpha_{k}\right|}+1\right) / 2=(4 k+1)(4 k+2) / 2=(4 k+1)(2 k+1) .
$$

On the other hand, each members of the sum, which are Kaczmarz function, take values \pm 1 . So, it can never be 0 and the value of such sum can not be less then 1 :

$$
\left|\sum_{j=1}^{n-2} \sum_{i=0}^{\left|\alpha_{k}\right|} \kappa_{i}(x)\right| \geq 1, \text { for all } x \in G
$$

It follows that

$$
\left|I V_{2}\right| \geq \frac{\Phi^{1 / 2 p}\left(2^{\left|\alpha_{k}\right|+1}\right)}{2^{\left|\alpha_{k}\right|+1}}
$$

Let $0<p<1 / 2$ and $n \in \mathbb{A}_{0,2}, 2^{\left|\alpha_{k}\right|}<n<2^{\left|\alpha_{k}\right|+1}$ and $x \in I_{2}\left(e_{0}+e_{1}\right)$. By combining (3.14,3.23) we have

$$
\begin{aligned}
& \left\|\sigma_{n}^{\kappa} F\right\|_{L_{p, \infty}}^{p} \\
\geq & \frac{c_{p} \Phi^{1 / 2}\left(2^{\left|\alpha_{k}\right|+1}\right)}{2^{p\left(\left|\alpha_{k}\right|+1\right)}} \mu\left\{x \in I_{2}\left(e_{0}+e_{1}\right):\left|\sigma_{n}^{\kappa} F\right| \geq \frac{c_{p} \Phi^{1 / 2 p}\left(2^{\left|\alpha_{k}\right|+1}\right)}{2^{\left|\alpha_{k}\right|+1}}\right\} \\
\geq & \frac{c_{p} \Phi^{1 / 2}\left(2^{\left|\alpha_{k}\right|+1}\right)}{2^{p\left(\left|\alpha_{k}\right|+1\right)}} \mu\left\{I_{2}\left(e_{0}+e_{1}\right)\right\} \geq \frac{c_{p} \Phi^{1 / 2}\left(2^{\left|\alpha_{k}\right|+1}\right)}{2^{p\left(\left|\alpha_{k}\right|+1\right)}} .
\end{aligned}
$$


Hence, by using (3.10) and (3.24) we get that

$$
\begin{aligned}
& \sum_{n=1}^{\infty} \frac{\left\|\sigma_{n}^{\kappa} F\right\|_{L_{p, \infty}}^{p}}{\Phi(n)} \geq \sum_{\left\{n \in \mathbb{A}_{0,2}: 2^{\left|\alpha_{k}\right|}\right.} \sum_{\{n<2} \frac{\left\|\sigma_{n}^{\kappa} F\right\|_{L_{p, \infty}}^{p}}{\Phi(n)} \\
\geq & \frac{1}{\Phi^{1 / 2}\left(2^{\left|\alpha_{k}\right|+1}\right)}\left\{\sum_{\left\{n \in \mathbb{A}_{0,2}:\left.2^{\left|\alpha_{k}\right|}\right|_{<n<2}\left|\alpha_{k}\right|+1\right.}\right\} \\
\geq & \frac{c_{p} 2^{(1-p)\left(\left|\alpha_{k}\right|+1\right)}}{\Phi^{1 / 2}\left(2^{\left|\alpha_{k}\right|+1}\right)} \rightarrow \infty, \text { as } k \rightarrow \infty .
\end{aligned}
$$

The proof is complete.

\section{REFERENCES}

[1] G.N. Agaev, N.Ya. Vilenkin, G.M. Dzhafarli, A.I. Rubinstein, Multiplicative systems of functions and harmonic analysis on 0-dimensional groups, "ELM" (Baku, USSR) (1981) (Russian).

[2] I. Blahota, On a norm inequality with respect to Vilenkin-like systems, Acta Math. Hungar. 89 (1-2) (2000), 15-27.

[3] I. Blahota, On the maximal value of Dirichlet and Fejér kernels with respect to the Vilenkin-like space, Publ. Math. 80 (3-4), (2000), 503-513.

[4] I. Blahota, K. Nagy, L. E. Persson, G. Tephnadze, A sharp boundedness result concerning some maximal operators of partial sums with respect to Vilenkin systems, Georgian Math., J., 26, (3) (2019), 351-360.

[5] G. Gát, On $(C, 1)$ summability of integrable functions with respect to the Walsh-Kaczmarz system, Studia Math. 130 (2) (1998), 135-148.

[6] G. Gát, Inverstigations of certain operators with respect to the Vilenkin sistem, Acta Math. Hung., 61 (1993), 131-149.

[7] G. Gát, U. Goginava, K. Nagy, On the Marcinkiewicz-Fejér means of double Fourier series with respect to the Walsh-Kaczmarz system, Studia Sci. Math. Hung., 46 (3) (2009), 399-421.

[8] U. Goginava, The maximal operator of the Fejér means of the character system of the p-series field in the Kaczmarz rearrangement, Publ. Math. Debrecen 71 (1-2) (2007), 43-55.

[9] U. Goginava, K. Nagy, On the maximal operator of Walsh-Kaczmarz-Fejér means, Czeh. Math. J. 61 (136) (2011) 673-686.

[10] B. Golubov, A. Efimov, V. Skvortsov, Walsh series and transformations, Dordrecht, Boston, London, 1991. Kluwer Acad. publ., 1991.

[11] K. Nagy, G. Tephnadze, Kaczmarz-Marcinkiewicz means and Hardy spaces, Acta math. Hung., 149 (2) (2016), 346-374.

[12] K. Nagy, G. Tephnadze, On the Walsh-Marcinkiewicz means on the Hardy space, Cent. Eur. J. Math., 12, 8 (2014), 1214-1228.

[13] K. Nagy, G. Tephnadze, Strong convergence theorem for Walsh-Marcinkiewicz means, Math. Inequal. Appl., 19, (1) (2016), 185-195.

[14] L.-E. Persson, G. Tephnadze, A sharp boundedness result concerning some maximal operators of Vilenkin-Fejér means, Mediterr. J. Math., 13 (4) (2016) 1841-1853.

[15] L.-E. Persson, G. Tephnadze, G. Tutberidze, On the boundedness of subsequences of Vilenkin-Fejér means on the martingale Hardy spaces, operators and matrices, 14 (1) (2020), 283-294.

[16] L.-E. Persson, G. Tephnadze, G. Tutberidze, P. Wall, Strong summability result of Vilenkin-Fejér means on bounded Vilenkin groups, Ukr. Math. J., (to appear).

[17] F. Schipp, Pointwise convergence of expansions with respect to certain product systems, Anal. Math. 2 (1976), 65-76.

[18] F. Schipp, W.R. Wade, P. Simon, J. Pál, Walsh Series. An Introduction to Dyadic Harmonic Analysis, Adam Hilger (Bristol-New York 1990). 
[19] P. Simon, Strong convergence of certain means with respect to the Walsh-Fourier series, Acta Math. Hung., 49 (1987) 425-431.

[20] P. Simon, On the Cesàro summability with respect to the Walsh-Kaczmarz system, J. Approx. Theory, 106 (2000) 249-261.

[21] P. Simon, Strong Convergence Theorem for Vilenkin-Fourier Series, J. Math. Anal. Appl., 245 (2000), 52-68.

[22] P. Simon, $(C, \alpha)$ summability of Walsh-Kaczmarz-Fourier series, J. Approx. Theory, 127 (2004) 39-60.

[23] P. Simon, Remarks on strong convergence with respect to the Walsh system, East Journal on Approx. 6 (2000), 261-276.

[24] V.A. Skvortsov, On Fourier series with respect to the Walsh-Kaczmarz system, Anal. Math., 7 (1981), 141-150.

[25] B. Smith, A strong convergence theorem for $H_{1}(T)$, Lecture Notes in Math., 995, Springer, Berlin, (1994), 169-173.

[26] A.A. S̆neider, On series with respect to the Walsh functions with monotone coefficients, Izv. Akad. Nauk SSSR Ser. Math. 12 (1948), 179-192.

[27] G. Tephnadze, On the maximal operators of Walsh-Kaczmarz-Fejér means, Period. Math. Hungar., 67 (1) (2013) 33-45.

[28] G. Tephnadze, Approximation by Walsh-Kaczmarz-Fejér means on the Hardy space, Acta Math. Scientia, (34B) (5) (2014) 1593-1602.

[29] G. Tephnadze, A note on the norm convergence by Vilenkin-Fejér means, Georgian Math. J., 21 (4) (2014), 511-517.

[30] G. Tephnadze, Strong convergence theorem for Walsh-Fejér means, Acta Math. Hungar., 142 (1) (2014) 244-259.

[31] G. Tutberidze, A note on the strong convergence of partial sums with respect to Vilenkin system, J. Contemp. Math. Anal., 54, 6 (2019) 319-324.

[32] W.S. Young, On the a.e converence of Walsh-Kaczmarz-Fourier series, Proc. Amer. Math. Soc., 44 (1974), 353-358.

[33] F. Weisz, Martingale Hardy spaces and their applications in Fourier Analysis, Springer, BerlinHeidelberg-New York, 1994.

[34] F. Weisz, Summability of multi-dimensional Fourier series and Hardy space, Kluwer Academic, Dordrecht, 2002.

[35] F. Weisz, - -summability of Fourier series, Acta Math. Hungar. 103 (2004), 139-176.

N. Gogolashvili, The University of Georgia, School of Science and Technology, 77a Merab Kostava St, Tbilisi, 0128, Georgia

E-mail address: nata.gogolashvili@gmail.com

K. Nagy, Institute of Mathematics and Computer Sciences, University of Nyíregyháza, P.O. Box 166, NyíregyházA, H-4400 Hungary

E-mail address: nagy.karoly@nye.hu

G. Tephnadze, The University of Georgia, School of Science and Technology, 77a Merab Kostava St, Tbilisi, 0128, Georgia

E-mail address: g.tephnadze@ug.edu.ge 